\title{
Cryptococcosis: an unusual cause of endobronchial obstruction
}

\author{
P. Mahida, R. Morar, A. Goolam Mahomed, E. Song, J.P. Tissandie, C. Feldman
}

Cryptococcosis: an unusual cause of endobronchial obstruction. P. Mahida, R. Morar, A. Goolam Mahomed, E. Song, J.P. Tissandie, C. Feldman. (CERS Journals Ltd 1996. ABSTRACT: We report the case of a 43 year old male patient, with normal immune function, who presented with right middle and lower lobe collapse.

At bronchoscopy, a white lobulated lesion was seen, completely obstructing the origin of bronchus intermedius. Bronchial washings and biopsy of the lesion demonstrated cryptococcal organisms.

The patient responded clinically and radiologically to amphotericin B and flucytosine; however, repeat bronchoscopy revealed only partial resolution of the endobronchial lesion.

Eur Respir J., 1996, 9, 837-839.
Dept of Medicine, Hillbrow Hospital and University of the Witwatersrand, Johannesburg, South Africa.

Correspondence: C. Feldman, Dept of Medicine, University of Witwatersrand, Medical School, York Road, Parktown 2193, Johannesburg, South Africa

Keywords: Amphotericin B, Cryptococcus neoformans, endobronchial obstruction, itraconazole

Received: April 101995

Accepted after revision November 121995
Pulmonary cryptococcosis usually presents with one or more dense parenchymal lung lesions and less commonly with pleural effusions, hilar lymphadenopathy and cavitary lesions [1].

Cryptococcosis presenting as an endobronchial lesion causing volume loss in the lung or lung collapse has only rarely been described $[2,3]$.

\section{Case report}

A 43 year old male patient presented with a 2 month history of cough productive of white sputum, progressive dyspnoea and mild weight loss. He had no pleuritic pain. He worked as a gardener and smoked 20 cigarettes $\cdot$ day $^{-1}$.

Physical examination revealed, initially, an alert and orientated patient. He appeared wasted, had an oral temperature of $38^{\circ} \mathrm{C}$ and no lymphadenopathy. He had a respiratory rate of 20 breaths $\cdot \mathrm{min}^{-1}$, the trachea was deviated to the right, and there was clinical evidence of right middle and lower lobe collapse.

Initial investigations revealed a leucocyte count of $8.7 \times 10^{9}$ cells $\cdot \mathrm{L}^{-1}$ and normal arterial blood gases on room air. Chest roentgenogram showed right middle and lower lobe collapse (fig. 1). A computerized tomographic (CT) scan of the chest showed no parenchymal lung lesion but there was evidence of narrowing of the bronchus intermedius in association with right middle and lower lobe collapse. Sputum microbiology, Ziehl-Neelsen and cytology and blood cultures were negative.

Fibreoptic bronchoscopy was performed under local anaesthesia and revealed a white lobulated endobronchial lesion obstructing the origin of bronchus intermedius, which was thought by the bronchoscopist to be a malignant lesion.
Bronchial washings and a biopsy of this lesion showed Cryptococcus neoformans organisms. Unfortunately, as the organisms did not grow on further culture, it was not possible to perform serotyping. Tests for human immunodeficiency virus by means of enzyme-linked immunosorbent assay (ELISA) as well as detection of p24 antigen were negative. Total lymphocyte count, CD4 and CD8 counts, and the CD4/CD8 ratio were normal. Skin tests for anergy were not performed. Immunoglobulins (Ig), including IgG subclasses, were normal. Following

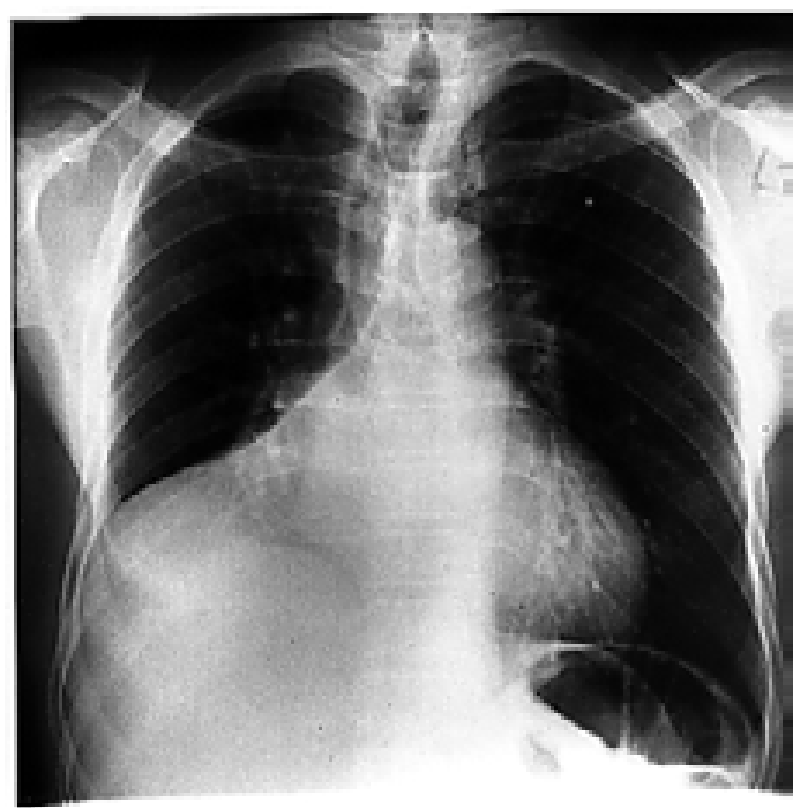

Fig. 1. - Chest radiograph on admission, showing right middle and lower lobe collapse. 
a test dose of $0.1 \mathrm{mg}$ amphotericin $\mathrm{B}$, given intravenously without systemic reaction, the patient was commenced on $30 \mathrm{mg}$ amphotericin B daily intravenously.

On the 10th day following admission (5 days after commencing amphotericin B therapy), the patient complained of mild headaches and subsequently became confused and unco-operative. He had no focal neurological deficit. A CT scan of the brain was normal and cerebrospinal fluid examination (performed only at the time of neurological involvement) revealed 35 lymphocytes $\cdot \mathrm{mm}^{-3}$ no neutrophils, normal biochemistry and two cryptococcal organisms. Flucytosine was added to his treatment. Approximately 8 days following the neurological complication, the patient improved, becoming orientated and co-operative.

Chest roentgenogram was repeated 3 weeks following institution of amphotericin B therapy and showed significant radiographic resolution of the right middle and lower lobe collapse (fig. 2). At this stage, the patient had received $750 \mathrm{mg}$ of amphotericin B therapy. A repeat cerebrospinal fluid examination was normal.

A repeat fibreoptic bronchoscopy was performed after the patient had received a total of 2,000 $\mathrm{mg}$ of amphotericin B therapy and showed only partial resolution of the endobronchial lesion. At this stage, it was unclear how the patient should be managed further. Apparent therapeutic options included continuation of medical therapy, surgical excision or more specialized techniques, such as laser therapy. The decision as to further therapy was taken by the patient, who refused any form of surgical intervention. The patient was discharged on flucytosine therapy.

On discharge from hospital, the patient was mentally normal and the repeat chest roentgenogram, although improved, still showed evidence of residual right middle lobe collapse. After 2 months of flucytosine therapy,

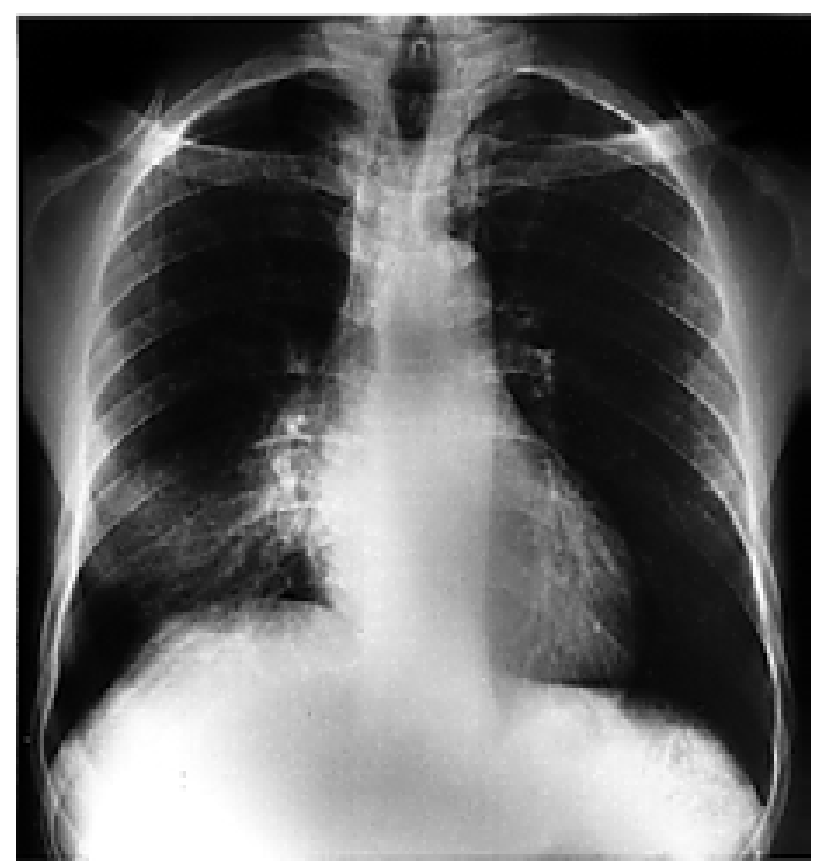

Fig. 2. - Chest radiograph after the patient had received $750 \mathrm{mg}$ of amphotericin B, showing significant resolution of the right middle and lower lobe collapse with no further resolution of the chest roentgenogram, the therapy was changed to itraconazole. Following 5 months of itraconazole therapy, the chest roentgenogram began to show evidence of additional resolution of the right middle lobe collapse. The patient declined a further fibreoptic bronchoscopy. Seven months following itraconazole therapy, oblique hilar tomography showed apparent complete resolution of the endobronchial obstruction. The current chest roentgenogram still shows mild residual right middle lobe collapse.

\section{Discussion}

Although pulmonary cryptococcosis occurs commonly as an opportunistic infection in immunocompromised patients, it is well recognized that it also occurs frequently in the lungs of normal individuals [1, 4]. We could find no evidence of immunocompromise in our patient, despite a number of investigations. Pulmonary cryptococcosis may present in a number of ways but is commonly shown radiologically as solid mass lesions [1]. In this respect, it may well be mistaken for a lung carcinoma and particularly so if there is associated lung collapse, as occurred in our patient. In immunocompromised patients, it may present additionally with some unusual features, including the presence of cavitation, pleural effusions and associated lymphadenopathy.

Endobronchial cryptococcosis causing complete lung collapse has, to the best of our knowledge, only been described once previously [2]. This patient had complete left lung collapse, and bronchoscopy showed a mass lesion totally occluding the left lower lobe bronchus and partially occluding the left upper lobe bronchus [2]. A patient with right upper lobe consolidation and minor volume loss has also been described [3] and at bronchoscopy, this patient had a gelatinous mass at the orifice of the right upper lobe bronchus [3].

Two patients with endobronchial cryptococcal infection without lung collapse have also been described [5, $6]$. One was a patient with right middle lobe consolidation, in whom bronchoscopy showed a haemorrhagic lesion covering the carina causing partial obstruction of the right and left main bronchi [5]. The other was a patient with a mediastinal mass, with infiltration of the trachea and the right and left main bronchi [6].

There appears to be no evidence from the literature that presentation with an endobronchial lesion is associated with immunocompromise. However, of the above patients, the immune status of three patients was unknown and one patient demonstrated skin anergy on presentation, which improved on therapy.

This patient presented to us with a long-term management dilemma. Although we had discussed the use of long-term medical therapy, specialized techniques, such as laser therapy or even surgical excision, the decision was made for us by the patient who refused any interventional therapy. The significant improvement in the patient on long-term medical therapy suggests that this is an acceptable alternative. This case report highlights the fact that cryptococcal infection of the lung, although rare, may mimic radiologically and bronchoscopically the features of lung carcinoma and needs to be considered in the differential diagnosis of endobronchial lesions. 


\section{References}

1. Seaton A Seaton D, Leitch A. Fungal diseases. In: Seaton A, Seaton D, Leitch A, eds. Crofton and Douglas's Respiratory Diseases: Oxford; Blackwell Scientific Publications, 1989; pp. 448-475.

2. Carter EA, Henderson DW, McBride J, Sage MR. Case report. Complete lung collapse: an unusual manifestation of pulmonary cryptococcosis. Clin Radiol 1992; 46: 292294.
3. Long RF, Berens SV, Shambhag GR. Case reports. An unusual manifestation of pulmonary cryptococcosis. $\mathrm{Br}$ J Radiol 1972; 45: 757-759.

4. Manson-Bahr PEC, Bell DR. Diseases caused by fungi. In: Mansons Tropical Diseases. 19th edn. Section VI. London; Bailliere Tindall, 1987; 714-716.

5. Town G, Seeman R. Pulmonary cryptococcosis: a report of two cases and review of the literature. NZ Med J 1985; 98: 894-895.

6. Sinha P, Naik KG, Bhagwat GP. Mediastinal cryptococcoma. Thorax 1978: 33: 657-659. 